\title{
Computer simulation of the sheath and the adjacent plasma in the presence of a plasma source
}

E.G. Shustin ${ }^{\text {a, c }}$,V.P. Tarakanov ${ }^{\text {b, c }}$, K. Ronald ${ }^{\mathrm{d}}$

${ }^{a}$ V.A. Kotelnikov Institute of Radio Engineering and Electronics of RAS, Fryazino Branch, Fryazino, Russia

${ }^{b}$ Joint Institute of High Temperatures of RAS, Moscow, Russia

${ }^{c}$ National Research Nuclear University MEPhI, Moscow, Russia

${ }^{\mathrm{d}}$ SUPA and the Department of Physics, University of Strathclyde, Glasgow, G4 0NG Scotland, United Kingdom

\begin{abstract}
A model is constructed allowing computer simulations of the near-wall area of a planar plasma sheet in conditions where the steady state of the plasma is supported by the production of charged particles in a region removed from the wall. Calculations have revealed variation in the energy distribution of the electrons in both time and spatially over the sheet width (cooling the electronic component) due to absorption of fast electrons at the walls bounding the plasma volume. It is shown that the plasma density profile across the sheet width has an abrupt decrease at the boundary of the region of plasma regulation. Thus the standard concepts of the potential and plasma density distributions in the sheath and presheath based on the assumption of a stable energy distribution for the electrons in the presheath yields inaccurate results for the plasma sheet where the ionization source is remote from the wall.
\end{abstract}

Keywords: Low temperature plasma; computer simulation; plasma sheath; energy distribution

\section{Introduction}

In our paper [1] results and analysis are presented of the effect of charging of a dielectric surface due to the impact of plasma particles on a structure consisting of a microwire on an insulating substrate which is set to a fixed potential which extracts 
ions. This problem was considered to address interest in obtaining a uniform profile of micro- and nanostructures of metal or semiconductor on an insulator by means of ion etching. To allow clear identification of the effects a rather simple model was chosen: collisionless plasma homogeneously filling the space in front of a wall. In these simulations we could observe only transient phenomena when supplying the potential to the microwire, because the timeframe of the problem was limited by plasma exhaustion due to the charged particles leaving the simulation volume, and being annihilated on interaction with the wall. Nevertheless, formation of an electrostatic lens was evident, accompanied with dielectric charging near the microwire. Thus there existed a heterogeneity of the profile of the ion flow close to the microwire, increasing over time, that would lead to undesirable heterogeneity of the etching rate near any such microwire in a real process.

In [1] the assumption was made that it is possible to remove or reduce the undesirable effect of increasing non-uniformity of the sheath ion beam by means of pulse modulation of the conductor potential. To investigate the processes associated with a pulsed potential on the conductor it is necessary to design a model which eliminates the constraint associated with plasma depletion.

The main goal of the present work is the development of such a model. The testing of the model was performed on a simplified configuration of a plasma-wall system that corresponds to that of a beam excited plasma in a plane geometry with regulation of the initial plasma parameters near surfaces which absorb the plasma particles colliding with them. Only after such a precondition is achieved will it be possible to investigate the dynamics of processes subject to pulsed potentials applied to the microwire. We believe that the results of these tests are of independent interest because they provide additional insight into the structure and behavior of the near-wall plasma in the presence of a remote source of ionization. 
Although the properties of the plasma sheath have been studied in detail and are often thought to be well known, in this paper we show that the standard concept based on the assumption of a constant energy distribution of the electrons in the pre-sheath, when applied to a plasma with an ionisation source remote from the walls, yields inaccurate results.

The main features of the plasma layer near the wall were revealed by I. Langmuir in his well-known kinetic analysis of the column of a low pressure discharge [2]. D. Bohm's analysis [3] gave formulation to the existence condition for a plasma sheath.

Detailed investigations of the sheath and pre sheath were conducted over a period spanning 80-90 years (see the review [4]). However theoretical investigation of the problem of the distribution of the charged particles in the plasma sheath near a material wall taking into account the influence of collisions, variation in the electron distribution function (from the Maxwellian assumed in the earliest analysis) and other factors remain an active area of research [5-7].

Let us recount the main properties of a near-wall plasma layer [4].

Loss of charged particles from the plasma to a wall due to ambipolar diffusion establishes a positive floating potential in the plasma relative to the wall. A sheath of positive space charge shields the wall. The sheath thickness is characterized by the local electron Debye length. A necessary condition for forming a stationary sheath is the Bohm criterion that demands ions reach the sheath area with a velocity not lower than the velocity of ion sound waves. If one biases the wall with a negative potential of a value exceeding $k T_{e}$ there are practically no electrons in the sheath, and it behaves according to the well-known Child-Langmuir law.

It is relevant to note that a more generally valid analysis of the structure of the plasma column bounded with a material wall should take into account ionization in the 
column, and such analysis has been reported [8-12]. Experimentally the structure of the plasma sheath and presheath was studied, for example in [13].

Application of numerical methods for the analysis of the structure and properties of the wall-plasma interface began in 1960s (The historical overview and the analysis of the current state of this research can be found in [14]). For studying the stationary state of a plasma layer by $\mathrm{PiC}$ simulation it is necessary to introduce to the model a source of plasma particles compensating for the loss of these particles on the absorbing wall(s). In the majority of publications on this topic glow or high-frequency discharges are considered as the source of charged particles. Procassini et al [15] used a PIC code to investigate the sheath and presheath ${ }^{1}$ for plasma with a distributed source remote from the absorbing wall. This geometry in essence simulates the beam excited plasma. We shall return to this article for comparison with the analysis of our results.

So, relying on the specified ideas of the structure of the plasma in the vicinity of a wall we have developed a model allowing the study of transient processes and the stationary state of near-wall plasma which may be exploited to investigate the dynamics associated with pulsed bias on the plasma boundaries.

\section{The model}

In this work, as well as in [1], research is performed by the method of numerical simulation. The mathematical model consists of the Poisson equation and the PiC model for a plasma containing electrons and ions, the equations of motion and conservation laws, including the self-consistent electric fields associated with plasma macroparticles. Solution of this system of equations is carried out by the KARAT code described in earlier publications $[1,16-18]$, where it was applied for the study of similar problems.

\footnotetext{
${ }^{1}$ Usually the word "presheath» refers to the region near the sheath in which the acceleration of the ions to the ion sound speed takes place. However some authors use this term in a more general sense, see for example, [14]: presheath i.e. a quasi-neutral plasma region adjacent to the sheath. For the purposes of this article we use this more general definition of the term.
} 
For simplicity and clarity, we choose the 1D model: the planar sheet of plasma bounded with charge absorbing walls (i.e. a conductor) at ground potential. The implication of this boundary is that charges encountering the boundary are immediately neutralized by an unlimited charge source. However all components of each particles velocity and the field strength are taken into account in the calculations. The plasma parameters are defined initially as follows: the electron energy distribution function is Maxwellian; $N_{e}=10^{10} \mathrm{~cm}^{-3}, T_{e}=2.5-10 \mathrm{eV}, T_{i}=0$. (This rather high upper limit of the electron temperature is chosen to provide more particles in the Debye length and hence to obtain more accurate results from the calculation. It is also advantageous to make sure that the Debye length $\lambda_{D e}$ exceeds the mesh spacing to prevent electron numerical heating). At the chosen plasma parameters the Debye length $\lambda_{D e}$ is $0.02-0.05 \mathrm{~cm}$. In the standard concept of a presheath [4] its thickness is more than tens of Debye length, therefore the width of the simulation region is taken to be equal to $2 \mathrm{~cm}$, i.e. about 100 Debye lengths. The plasma is modelled by a sufficient number of particles, of the order of $10^{5}$, so that in the Debye length and moreover within a simulation grid cell there are many tens and hundreds of particles; this provides reliable simulation results. We used 400 cells over the sheet width. This resolution was determined by convergence testing, when further increase ceased to affect the calculation result. The calculations have shown that the approximate distribution of the particles over the sheet width is 4500 in the sheath $(0<\mathrm{Z}<0.15 \mathrm{~cm}) ; 39000$ in the bulk plasma $(0.15<\mathrm{Z}<0.85 \mathrm{~cm}) ; 16500$ in the regulation region $(0.85<\mathrm{Z}<1 \mathrm{~cm})$.

As the goal is research into the stationary state of the near-wall plasma, we need to include a plasma source in the model. Inclusion of an electron beam in the central region, which ionizes the gas at a rate which ultimately matches the loss rate and by that means keeps the plasma density constant (which of course is strictly consistent with some experiments), could be one method of meeting this requirement. Such a model 
would directly simulate known plasma sources with beam excited plasma [19-22], and is also connected with problems of beam plasma discharge (BPD) considered for example in $[17,18,23]$.

However including the walls required to emit and absorb this beam into this model would enforce a change of the numerical problem to at least 2 spatial dimensions, very substantially increasing the computational difficulty of the problem. Moreover it would also force us to study the influence of these additional wall interfaces on the processes immediately interesting to us and, thus, would compromise our ability to interpret the simulations regarding the properties of the transition sheet. Therefore, the model was simplified (see Fig.1).

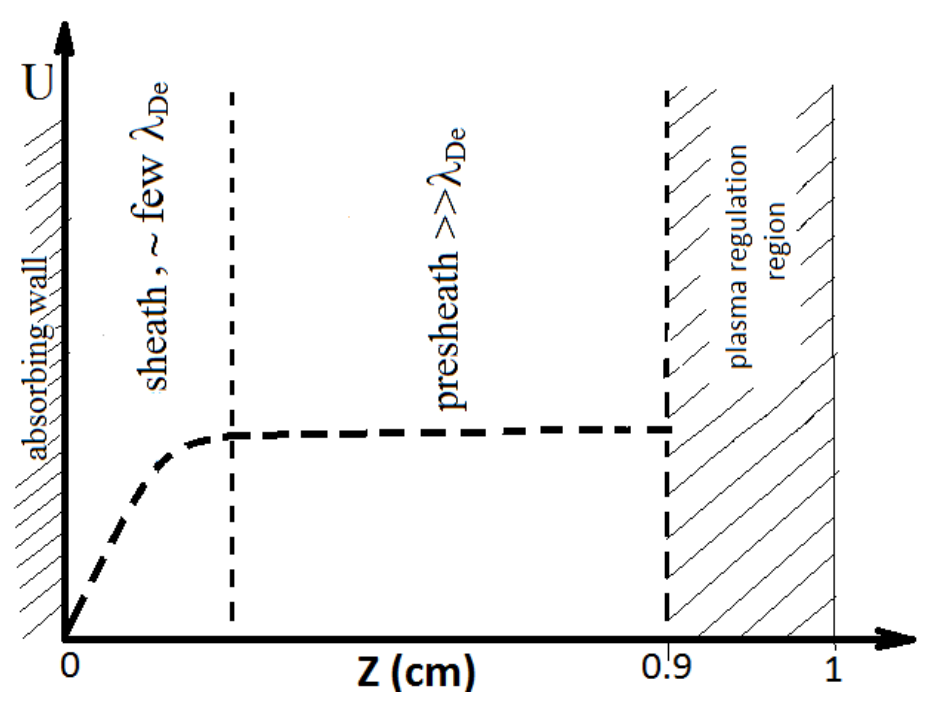

Fig. 1. Geometry of the model and qualitative view of the potential dependence vs distance, $\mathrm{z}=1 \mathrm{~cm}$ corresponds to the center of the symmetric $1 \mathrm{D}$ problem.

We select an artificial method for keeping the plasma density constant that is equivalent to the physical process from the point of view of understanding the sheath dynamics.

The algorithm is implemented as follows. At a certain region in the center of the symmetric simulation region $(0.9<Z<1.1)$ the density of the plasma electrons is monitored and when it decreases, electron-ion pairs are added with the same distribution 
in energy as the initial plasma particles. As the particles lost from the region of density moderation tend to be those with velocity aligned with the axis of the simulation, whilst the re-population is isotropic, a difference would develop between the EDF perpendicular and parallel to the simulation axis. To inhibit this undesired effect, although in the sheath and bulk plasma the plasma is collisionless, in the central region, where the plasma density is sustained, we introduce elastic collisions between the electrons with the heavy neutral gas with a sufficiently high frequency to ensure the plasma remains isotropic. The mean free path for these artificial conditions is on the order of the thickness of the central, density moderated region. As the energy exchange rate to the gas particles is weak, the electron energy is scarcely affected by these elastic collisions. Collisions between the ions and neutrals are not considered. Therefore, just as the addition of electron-ion pairs compensate for any decrease in electron density in this 'regulated' central region, these artificial collisions ensure uniformity of the plasma over all velocity components in the same region. This prevents the accumulation of electrons, reflected from the wall and having little velocity components normal to the wall. As will be shown, processes in the boundary sheet change the energy distribution of the electron components, even in the center of the plasma, despite these measures to inhibit the dissipation of the plasma.

Of course, this model does not describe the processes associated with the initiation of the plasma during pulse injection of an electron beam into a rarefied gas. The only transient phenomenon in our case is the process of forming of a near-wall layer, this includes the self-consistent evolution of the density distribution of the particles and the associated electric fields. But, as stated above, the goal of the immediate work (ultimately we wish to modulate the potential in microwires in future research) is the study of a stationary state of the plasma layer in the presence of a 
plasma source, and this state of the beam excited plasma is adequately described by the model.

The characteristics of beam excited plasmas were studied experimentally for cylindrical $[19,20]$ and plane $[21]$ geometries. In both cases a rather definite localization of the plasma region was shown, remote from the walls, in spite of the large mean free path of ions relative to the lateral dimension of the plasma chamber.

The paper [23] describes an experiment in which the area of production of plasma in the beam plasma discharge is localized and rather remote from the absorbing walls, and where the mean free path of the electrons significantly exceeds the transverse size of the chamber.

Thus, the model used in our work reflects qualitatively the reality of these physical experiments.

\section{The simulation results and discussion}

To evaluate the physical validity of the model and reveal a qualitative picture showing the creation of a stationary sheath, an artificial plasma with an ion mass $M=1000 m$ (where $m$ is the mass of an electron) was chosen for the first stage of the simulations. These simulations revealed new, nontrivial, effects with a rather small computational time requirement.

In fig. 2 the time evolution of the potential on the central plane for different initial temperatures of the electrons $\left(T_{e}=2.5 ; 5 ; 10 \mathrm{eV}\right)$ is shown. It is evident that in the beginning (after several transit times of the electrons through the sheet) the potential in the center reaches a values corresponding to the well-known formula for the floating potential

$U_{p}=T_{e} \ln (\pi m / 2 M) / 2 e$

(for $M / m=1000 U_{p} \sim 3 T_{e}$ ). However, the potential subsequently falls monotonically or with considerable oscillations) down to around $\sim 0.2 U_{p}$. The steady state is achieved 
after $1 \mu \mathrm{s}$. The steady state condition is confirmed also by an analysis of the dependencies of other integral parameters of the system, including total charge of the electrons and ions vs time and the temporal evolution of the spatial distribution of the ion density.

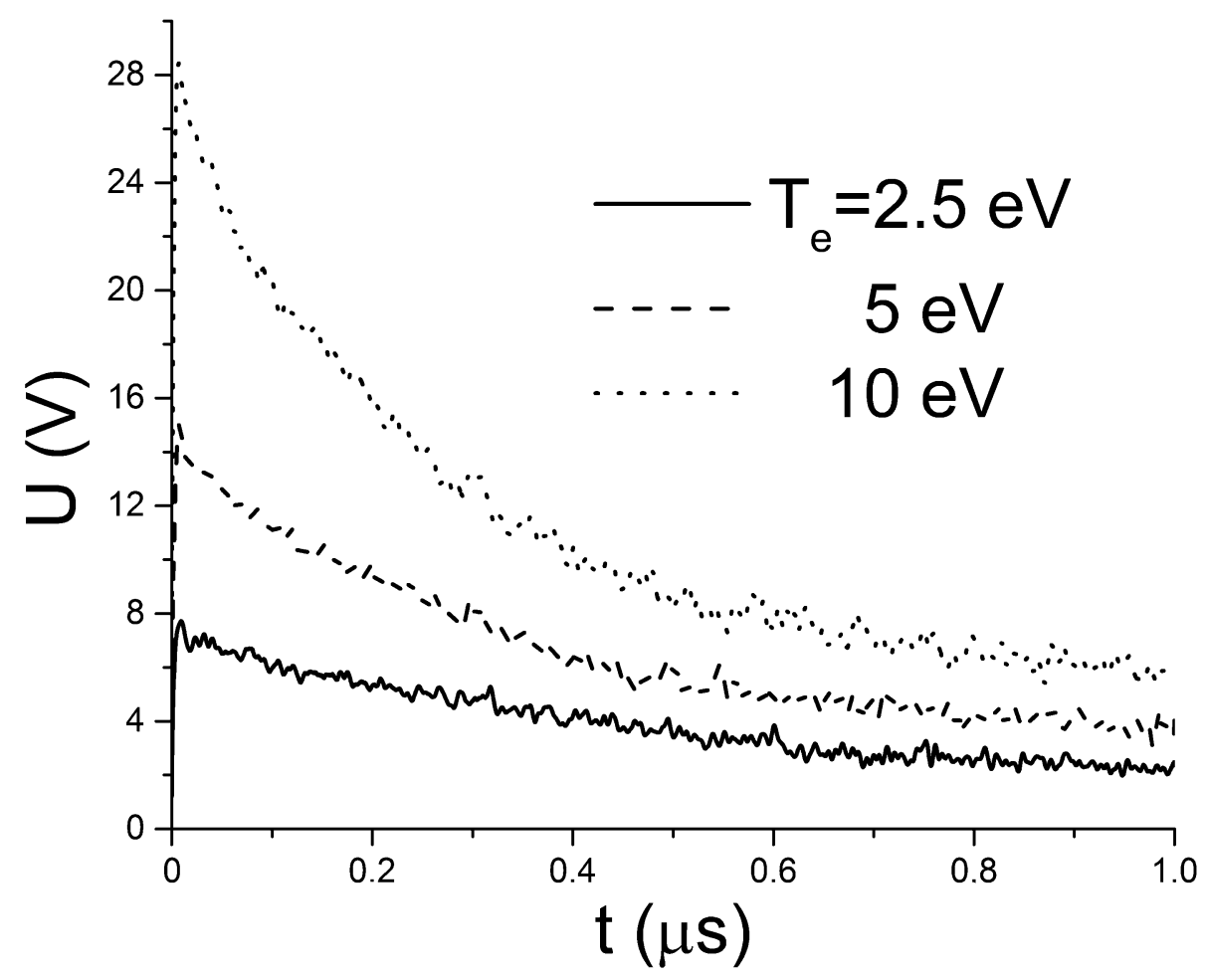

Fig. 2. Temporal evolution of the potential in the center of the simulation $(Z=1 \mathrm{~cm})$ for different electron temperatures $T_{e}$.

One can find an explanation for the observed voltage drop at the sheet from curves for the electron energy distribution at the center and near the wall (Fig. 3$)^{2}$. Despite continuous "feeding" of the central region with electrons with the initial temperature, the electron component of the plasma is considerably cooled due to the more rapid departure of the faster electrons to the walls, such that the distribution function changes over the sheet width. Let us recall that the standard ideas of the

\footnotetext{
${ }^{2}$ The electron energy distribution function (EEDF) $F(W, Z, t)$ is calculated over the particles in the vicinity of the point $Z$. The size of the region is taken to be $0.05 \mathrm{~cm}$, which is less than the characteristic spatial scale for variation of plasma parameters, but still involves hundreds of particles in the analysis.
} 
potential and plasma density distributions in a sheath and a presheath are based on a postulate that the electron energy distribution function (EEDF) remains isothermal and homogeneous in all area of quasi-neutrality.

As can be seen in Fig. 3, the simulated distribution functions after $1 \mu$ s are noticeably different in shape from the curves of the initial Maxwell distribution with the initial $T_{e}=10 \mathrm{eV}$. Recall, however, that the effect of electron scattering on ion-acoustic oscillations excited in the system can quickly thermalize the distribution function [24], if the sheet width is sufficiently large compared to the effective length of collisionless scattering. To estimate this effect we have performed calculations with a sheet width increased by a factor of 2 (see Section 4).

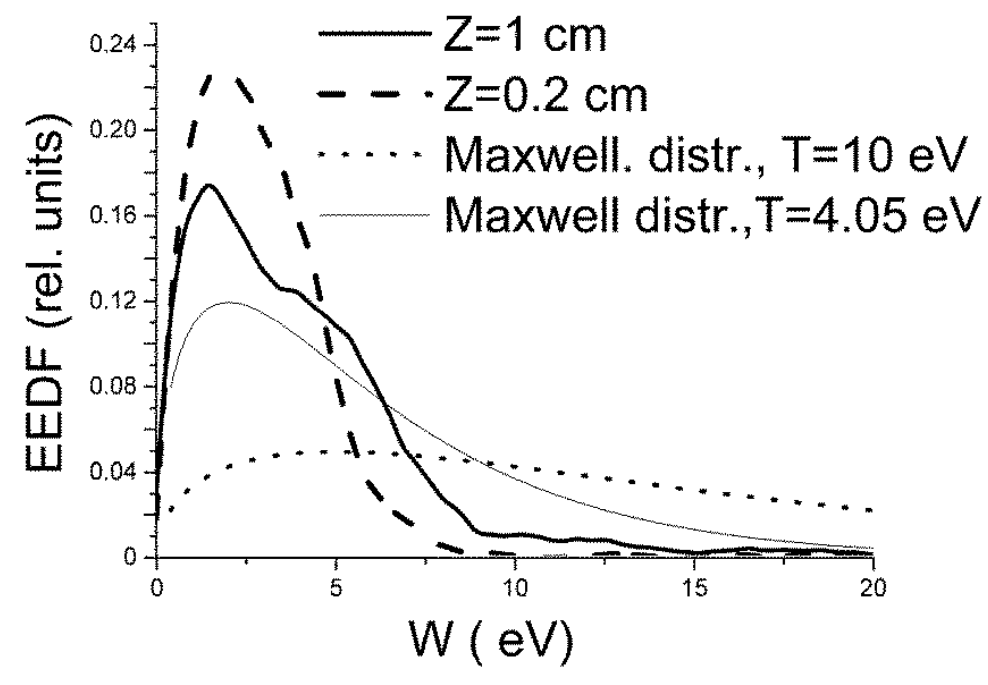

Fig. 3. Electron energy distributions at the sheet center and at a distance of $2 \mathbf{~ m m}$ from the wall after $1 \mu \mathrm{s}$ for an initial electron temperature of $10 \mathrm{eV}$. The Maxwellian distributions with $T_{e}=10 \mathrm{eV}$ (i.e. the distribution at $t=0$ ) and with $T_{e}=4.05 \mathrm{eV}$ are shown for comparison. Note the temperature at $\mathrm{Z}=1 \mathrm{~cm}$ has declined close to $4 \mathrm{eV}$ after $1 \mu \mathrm{s}$.

The results presented in fig. 4 showing profiles of the plasma particle densities across the sheet at $t=1 \mu$ s are also interesting. The figures reveal that the plasma density 
falls sharply from the charge formation boundary to $\sim 0.4$ of the predefined density in the center and then decreases more progressively to the sheet border. Note the rapid reduction of the plasma density from the center of the plasma sheet to the edge of the region in which the electron beam produces new plasma particles, is revealed experimentally in $[19,21]$ (for beam excited plasma) and in [23] (for beam plasma discharge).
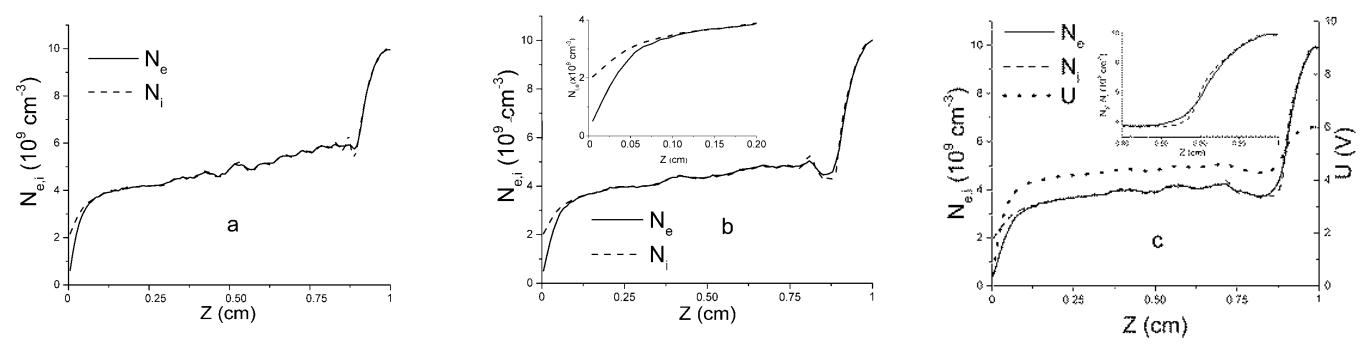

Fig. 4. Snapshots of profiles of electron $N_{e}$ and ion $N_{i}$ densities with ion mass $M=1000 m$, (a) $T_{e}=2.5 \mathrm{eV}$; (b) $5 \mathrm{eV}$; (c) $10 \mathrm{eV}$, at moment $t=1 \mu \mathrm{s}$. In fig. 4c the potential profile $U$ is also shown. Inset in fig. $4 \mathrm{~b}$ shows the density profiles near the wall; while in fig. 4c the inset shows the density profiles near the center of the sheet.

It is possible to notice a difference in the slopes of the curves $N_{e}(Z)$ in the bulk plasma for different initial electron temperatures. One must keep in mind, however, that the correct scale length to consider is not physical distance $Z$, but rather distance measured in $R_{D}$, which of course depends on temperature and varies spatially according to the change in $N_{e}$.

The reason for the existence of this area with reduced density becomes clear from the analysis of figures $4 \mathrm{c}$ and 5.

Fig. $4 \mathrm{C}$ shows that an electric field $E_{z}=d U / d Z$ is created at the central region due to loss of electrons. This field approaches its maximum on the boundary of the regulation region $(\mathrm{Z}=0.9 \mathrm{~cm})$, with a magnitude $\sim 10.6 \mathrm{~V} / \mathrm{cm}$ for initial condition $T_{e}=2.5$ $\mathrm{eV}, \sim 29 \mathrm{~V} / \mathrm{cm}$ for $T_{e}=5 \mathrm{eV}$ and $\sim 37 \mathrm{~V} / \mathrm{cm}$ for $T_{e}=10 \mathrm{eV}$. This field accelerates ions into 
the bulk plasma (see Fig. 5a). The ions are created at different points across the charge production region and therefore at a range of potentials associated with the region of their birth, thus a distribution of the ion velocities in the bulk plasma is observed. As the flow continuity condition is satisfied in the stationary stage: $N_{i} V_{i}=$ const, the ion density falls with growth of ion velocity. From figure $5 b$ one can see that the distribution of the electrons in the bulk plasma is obviously limited to some maximum velocity $V$ (fast electrons leave to the wall) and is almost symmetrical around $V=0$ due to counter flow of electrons below the critical threshold.
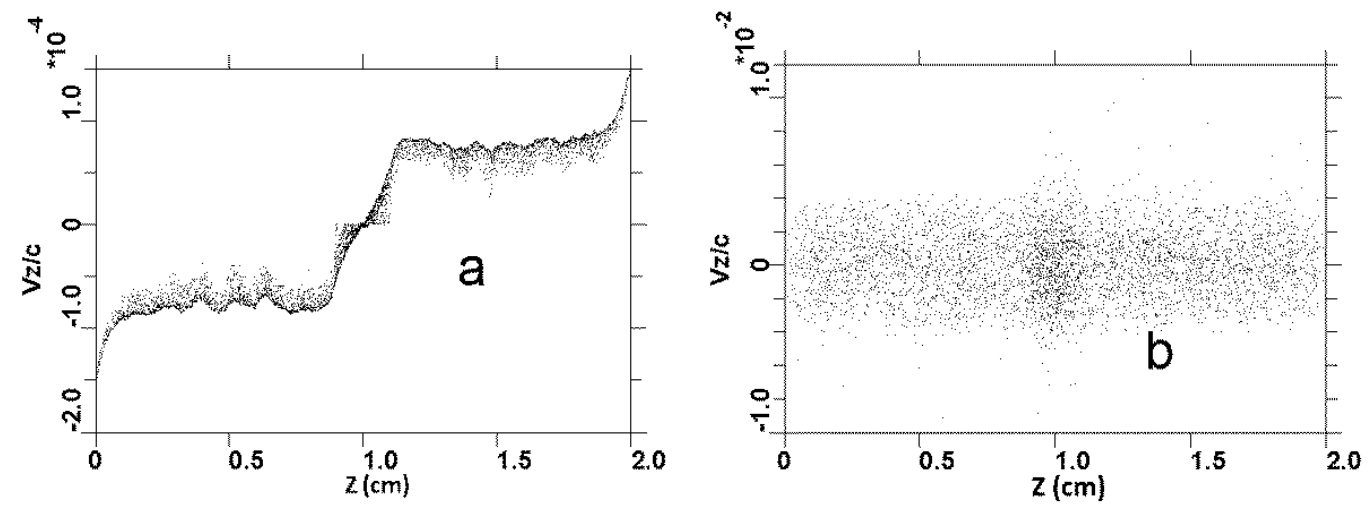

Fig. 5. Phase plane $\left(V_{z}, Z\right)$ of (a) ions and (b) electrons at $t=1 \mu \mathrm{s}, M=1000 m, T_{e}=10$ eV.

In the inset on Fig. 4b showing profiles of the plasma density at the wall in an expanded scale one can clearly see the plasma sheath where the plasma quasi-neutrality is broken. Its width is $\sim 0.5 \mathrm{~mm} \sim 5 R_{D}$ (here $R_{D}$ is the local Debye radius near the sheath boundary).

Let us remember that according to the Bohm criterion the initial speed of the ions at the sheath boundary has to exceed $\left(T_{e} / M_{i}\right)^{1 / 2}$, i.e. the ions must have energy > $T_{e} / 2$ as the condition for the formation of a stationary sheath. From the total voltage drop near the center and through the bulk plasma it is possible to determine the kinetic energy of the ions at the sheath boundary as $\sim 3 \mathrm{eV}$ (for initial $T_{e}=10 \mathrm{eV}$ ). Taking into 
account the effect of cooling of the electrons shown above, we see that the Bohm criterion is fulfilled.

Calculations were also carried out for ion masses of $M_{i}=5000 \mathrm{~m}$ and $M_{i}=25000 \mathrm{~m}$. In figures 6 and 7 the electron energy distributions and profiles of the electron density for these masses at $T_{e}=10 \mathrm{eV}$ and $t=2$ and $10 \mu \mathrm{s}$, respectively, are shown for comparison. One can see in Fig. 7b that the electron density profiles has converged after $5 \mu$ s (the differences from 5 to $10 \mu$ s does not exceed the inherent random fluctuations). The EEDF (shown in figure 6b) had substantively converged by $3 \mu \mathrm{s}$. Thus, the characteristic time required to establish a stable sheath for this mass of an ion is $5 \mu \mathrm{s}$.

As one would expect, the cooling effect on the electrons is weakened for these heavier ions, but nevertheless remains important, especially in the near-wall region.
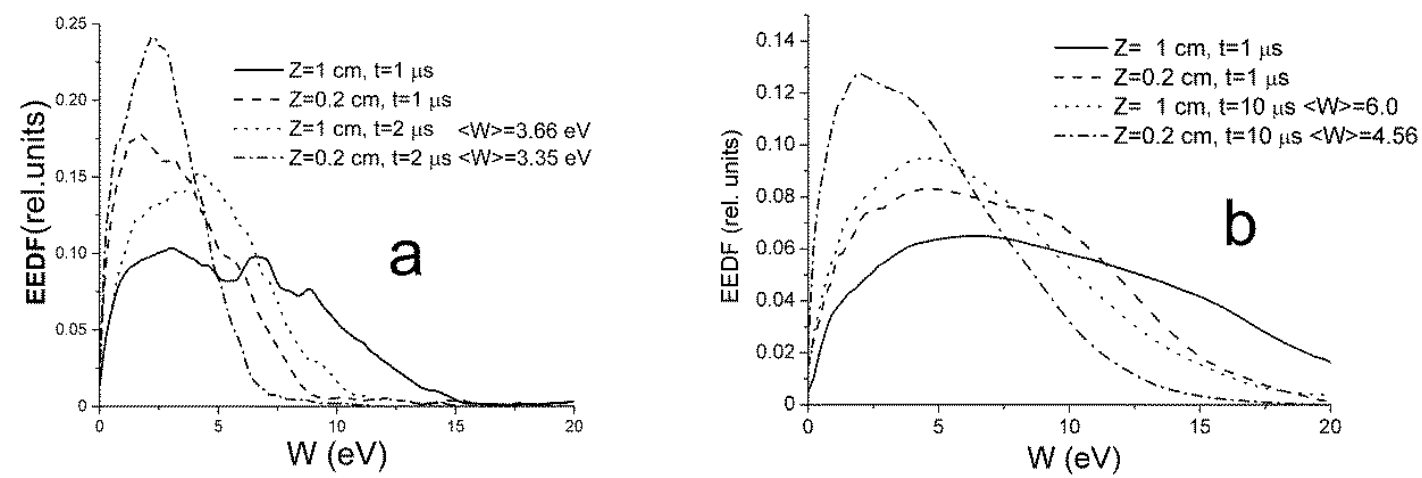

Fig. 6. Electron energy distributions for (a) $M_{i}=5000 \mathrm{~m}$ and (b) $M_{i}=25000 \mathrm{~m}$. The parameter $<W>$ corresponding to the mean electron energy at the center $(Z=1 \mathrm{~cm})$ and at $Z=0.2 \mathrm{~cm}$ during the stationary stage of the process is included in the legend. 

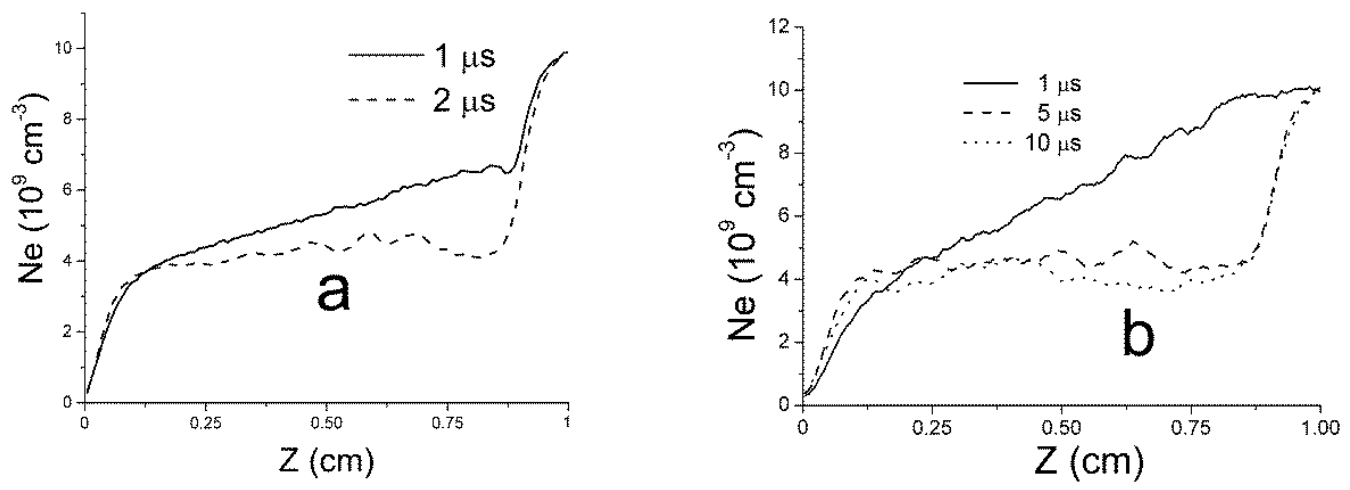

Fig. 7. Snapshots of profiles of electron density $\mathrm{Ne}(\mathrm{Z})$ for (a) $M_{i}=5000 \mathrm{~m}$ and (b) $M_{i}=25000 m$

Let us compare the results above with those of [15]. In this paper the problem of a near-wall collisionless plasma was calculated by the PiC method of simulation with an ionization source, distributed over the central part of the plasma sheet. The authors used an algorithm for the evolution of the system towards a state of equilibrium which differs from that used in the present paper. For numerical evaluation and for plotting of specimen results the authors used initial plasma parameters chosen to show a range of plasma conditions and for numerical efficiency: $k T e_{0}=50 \mathrm{eV} ; T_{e} / T_{i}$ in the range 0.25-2.0; $M / m$ in the range of 10-300; which differ from those which we have selected as we are interested in a low pressure discharge environment. For the settings used in [15] the electron distribution function tends to Maxwellian when approaching the wall due to the excitation of intense potential fluctuations. However, as with our calculations, a significant change of the electron distribution function (a decrease of the effective temperature) from the source region to the wall was shown. The potential profiles within the plasma sheet presented in [15] were qualitatively similar to those calculated in our work.

One can compare our results with those of the paper [8], where a static theory of a beam-generated plasma for beams partially or completely filling the space between two plane conducting walls was derived. These authors used the fluid model of the 
system and solved Poisson's equation with a predetermined electron temperature and a fixed rate of ion-electron pair generation. As the authors affirmed, "this theory can be used to predict the density and sheath height of such a plasma when the electron temperature is not too high". The sheath height, i.e. the voltage drop on the sheath calculated by this theory is in essence the value defined by formula (1) with a small modification dependent on the system geometry and the charged particle generation. As is shown above, the change of effective temperature in time and over space results in essential deviation from this formula.

\section{Estimation of the effects of elastic binary collisions and scattering}

To assess the role of collisions, we conducted an additional series of calculations by introducing into the model elastic collisions in the sheath and bulk plasma areas with atoms of the neutral gas, with a mean free path for the electrons of $0.2 \mathrm{~cm}$. The calculations were undertaken for the ion mass $M=1000 m$ for which the coefficient of energy exchange of electrons with neutrals is the largest among the ion masses used in the calculations presented in this paper. The calculations (see figure 8) have shown that the EEDF curves are scarcely affected by the collisions. The density profiles are practically identical to the ones shown in Fig. 4c. Obviously, inelastic collisions at $T_{e} \leq 10 \mathrm{eV}$ will have even less influence on the processes studied.

For qualitative estimation of the influence of collisionless scattering of electrons we have also carried out calculations with the same plasma conditions used for the results presented in Fig. 3, 4c, 5, but with twice the width of the plasma layer: $Z=4 \mathrm{~cm}$. The resulting electron energy distribution functions are shown in Fig. 9. It is seen that the cooling effect of electrons and the variation of the distribution functions in the bulk plasma is preserved, but the distribution in the center is noticeably closer to Maxwellian. 


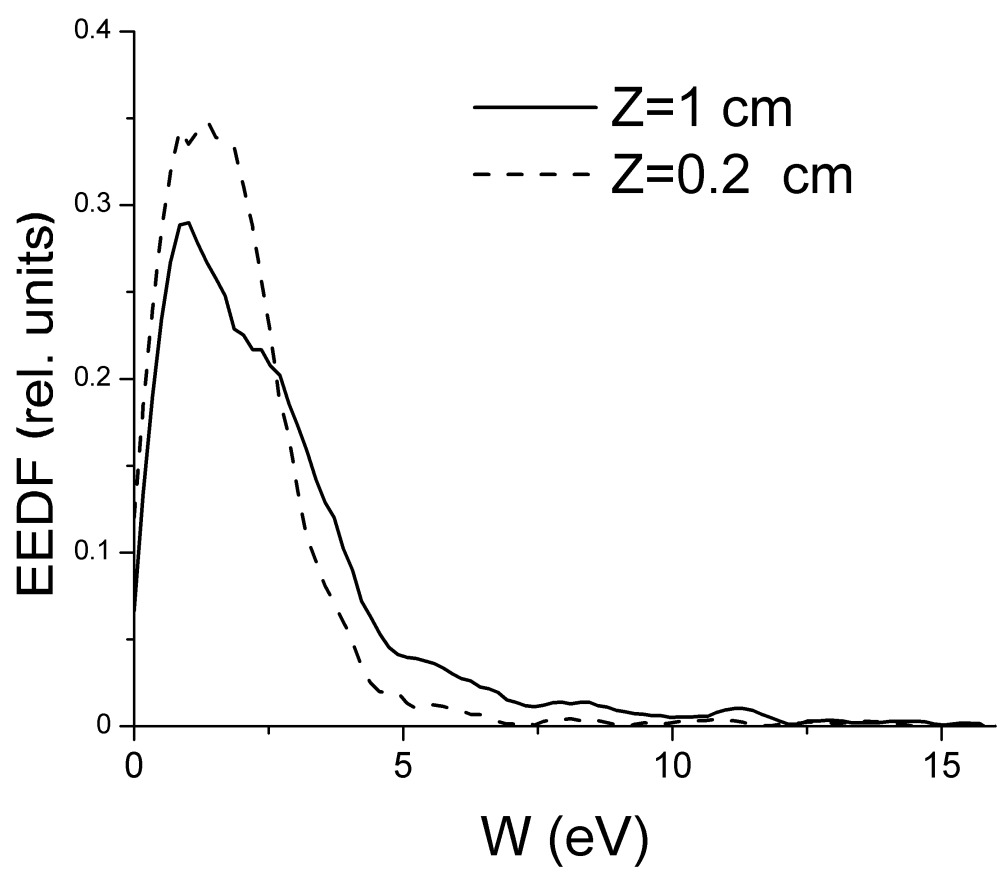

Fig. 8. EEDF of electron and ion densities in the plasma sheet at the moment $t=1 \mu \mathrm{s}$ with electron-neutral collisions included in the simulation.

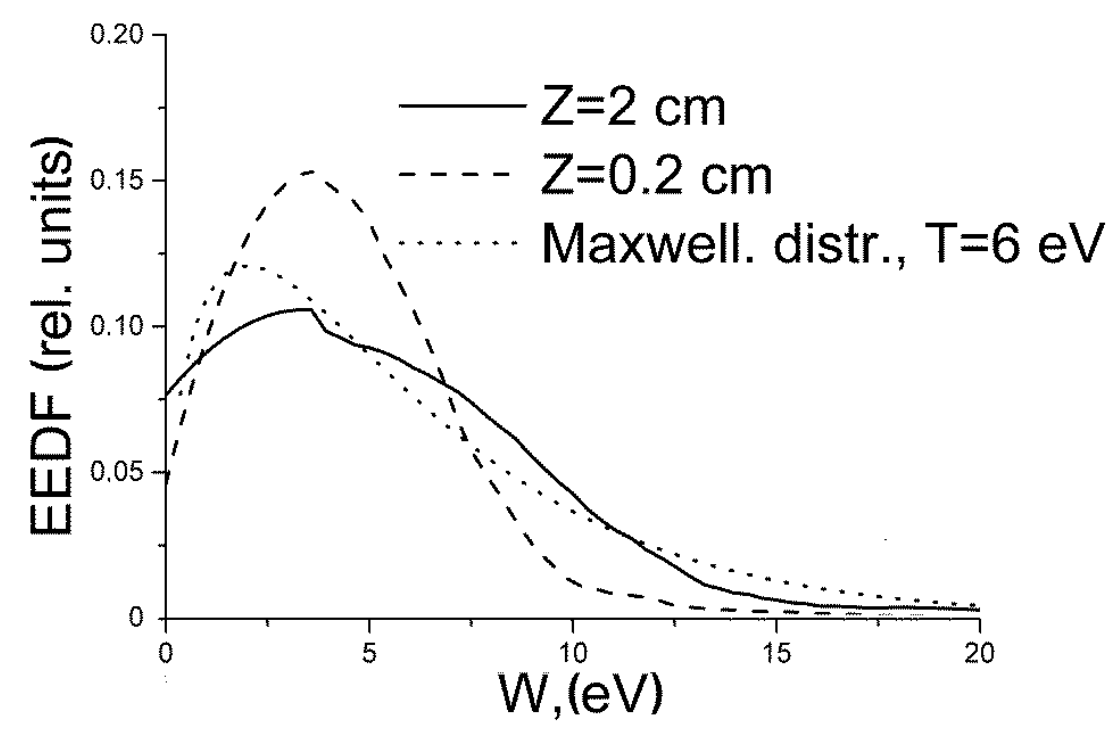

Fig. 9. EEDF at the centre $(Z=2 \mathrm{~cm})$ and near the edge $(Z=0.2 \mathrm{~cm})$ at the moment $t=1 \mu$ for a simulation with twice the transverse dimensions of those used to generate figures 3-5. Also shown is a comparison to the Maxwellian at $6 \mathrm{eV}$. 


\section{Conclusion.}

In this work a model is constructed allowing computer simulation of a near-wall plasma sheet in conditions when stable background plasma is supported against losses from the simulation volume by its regulation in the central part of the sheet. Although, ostensibly, the structure of the wall-plasma interface has been investigated theoretically in detail, the present simulations using a stable plasma source permitting self-consistent calculation of the dynamics of the plasma sheath formation have allowed us to develop an understanding of the impact the plasma edge has on the electron temperature in the bulk plasma (this cooling effect has also been reported in [15], but was not hitherto considered in the theory). This effect is associated with the marked change of electron energy distribution in time and on location in the sheet (cooling of the electronic component of the plasma) due to absorption of fast electrons at the walls bounding the plasma volume. It is relevant to recall that the theory of a collisionless sheath postulates an unvarying EEDF for electrons in the presheath and the bulk plasma. Cooling of the plasma leads to a significantly smaller stationary floating plasma potential than follows from the classical theory. Here it is necessary to emphasize that the observed effect is not connected with the influence of inelastic collisions which can also lead to depletion of the high-energy "tail" of the electron energy distribution.

Analysis of the distribution of the plasma density over the sheet cross-section showed an abrupt decrease in the particle density at the boundary of the charge production region as an unexpected result. The analysis of the potential distribution across the sheet and the phase planes $\left(\mathrm{V}_{\mathrm{z}}, \mathrm{Z}\right)$ showed that the reason for this drop is the potential jump at this boundary due to the faster drift of electrons from the center compared with ions. This potential difference creates a flow of ions from the center to the bulk plasma, and due to the growth of the ion speed, their density decreases. The 
electron component of the plasma is formed by a flow of thermal electrons from the center and by the reflected return flow from the wall.

In this stage of our investigation we applied a rather simple model of plasma with an abruptly bounded region of plasma production and a rather small size for the calculation area. This revealed the main qualitative effects with clarity and for a moderate calculation time for a wide range of parameter space. We plan to estimate the effects of extending the zone between the plasma production area and the wall in a future development of this study.

Let us recall that the motivation for this investigation is the need to create a model allowing the investigation of non-stationary processes under pulsed potential conditions at a wall or some component in a wall (microwire). The model outlined and validated herein provides the basis on which these further simulations, with important impact for applications, may be undertaken.

\section{Acknowledgements}

The authors are grateful to Prof. P. S. Strelkov, A.M. Prokhorov Institute of General Physics of RAS, Russia for valuable remarks on the text of the report.

This work was supported, in part, by the Russian Foundation for Basic Research (project no. 14-08-00143) and by the Competitiveness Program of NRNU MEPhI.

\section{References}

[1] Tarakanov VP, Shustin EG. Vacuum 2015; 113:59

[2] Langmuir I. Phys. Rev. 1929;.33:954

[3] Bohm D. The Characteristics of Electrical Discharges in Magnetic Fields. ed. A Guthry and R K Wakerling (New York: MacGraw-Hill) 1949, ch 3: 77

[4] Rieman K.-U. J. Phys. D: Appl. Phys 1991; 24:492

[5] Sternberg N, Godyak V. IEEE Trans. on Plasma Sci 2007; 35:1341 
[6] Riemann K-U. Plasma Sources Sci. Technol. 2009; 18:014006; Ibid., 014007

[7] Hatami M. Physics of Plasmas 2015; 22:023506

[8] Dunn DA, Self SA. Journal of Applied Physics, 1964 35: 113

[9] Self SA, Ewald HN. Phys. Fluids 1966; 9:2486

[10] Kamran M, Kuhn S, Tskhakaya DD, Khan M. Plasma Physics 2013; 79, pt 2, 169

[11] Mizrahi JHP. Physics of Plasmas 2014; 21:063509

[12] Valentini H-B, Kaiser D. Plasma Sources Sci. Technol. 2014; 23:015004

[13] Oksuz L, Hershkowitz N. Plasma Sources Sci. Technol 2005;14:201

[14] Robertson S. Plasma Phys. Control. Fusion 2013; 55: 093001

[15] Procassini RJ, Birdsall CK,. Morse EC. Physics of Fluids 1990; B2: 3191

[16] Tarakanov VP. User's manual for code KARAT. Springfield, Va: Berkeley, Research Associates Inc; 1992.

[17] Tarakanov VP; Shustin EG. Plasma Physics Reports 2007; 33:151

[18] Klykov IL; Tarakanov VP; Shustin EG. Plasma Physics Reports 2012; 38: 263

[19] Hedvall P. Journal of Applied Physics, 1962 33: 2426

[20] Chalmers, ID. Phelps, ADR., de Ste Croix, G. IEEE International Symposium on Electrical Insulation, 1992 p. 21

[21] Meger RA, Blackwell DD, Fernsler RF, Lampe M, Leonhardt D, Manheimer WM et al. Phys. Plasmas 2001; 8:2558

[22] Leonhardt D, Walton SG, and. Fernsler RF. Phys. Plasmas 2007; 14:057103

[23] Isaev NV, Chmil’ AI, Shustin EG. Plasma Physics Reports, 2004; 30: 263

[24] B. S. D. Baalrud, J. D. Callen, and C. C. Hegna, Phys. Rev. Lett. 2009; 102, 245005 